Old Dominion University

ODU Digital Commons

\title{
Stability and Sensitivity Measures for Solutions in Complex, Intelligent, Adaptive and Autonomous Systems
}

Andreas Tolk

Old Dominion University, atolk@odu.edu

Follow this and additional works at: https://digitalcommons.odu.edu/msve_fac_pubs

Part of the Computer and Systems Architecture Commons, and the Systems and Communications Commons

\section{Original Publication Citation}

Tolk, A. (2016). Stability and sensitivity measures for solutions in complex, intelligent, adaptive and autonomous systems. Paper presented at the Proceedings of the Modeling and Simulation of Complexity in Intelligent, Adaptive and Autonomous Systems 2016 (MSCIAAS 2016) and Space Simulation for Planetary Space Exploration (SPACE 2016), Pasadena, California, April 3-6, 2016. https://dl.acm.org/ citation.cfm?id=2962671

This Conference Paper is brought to you for free and open access by the Computational Modeling and Simulation Engineering at ODU Digital Commons. It has been accepted for inclusion in Computational Modeling and Simulation Engineering Faculty Publications by an authorized administrator of ODU Digital Commons. For more information, please contact digitalcommons@odu.edu. 


\title{
Stability and Sensitivity Measures for Solutions in Complex, Intelligent, Adaptive and Autonomous Systems
}

\author{
Andreas Tolk \\ The MITRE Corporation \\ 903 Enterprise Pkwy \#200 \\ Hampton, VA 23666 \\ atolk@mitre.org
}

\begin{abstract}
Simulation has become a pivotal tool for the design, analysis, and control of complex, intelligent, adaptive and autonomous systems and its components. However, due to the nature of these systems, traditional evaluation practices are often not sufficient. As the components follow adaptive rules, the cumulative events often exploit bifurcation enabling events, leading to clusters of solutions that do not follow the usual rules for standard distributed events. When using simulation for design, analysis, and control of such systems, the evaluation needs to be richer, applying bifurcation and cluster analysis to understand the distribution, applying factor analysis to understand the important factors for the necessary sensitivity analysis, and take not only point estimates for the solution and the sensitivity analysis into account, but contact a statistical stability analysis. The full exploitation of gaining numerical insights into the dynamic behavior and its deviations is needed. This paper introduces the pitfalls and recommends applicable methods and heuristics.
\end{abstract}

\section{Author Keywords}

Bifurcation; Cluster Analysis; Factor Analysis; Sensitivity; Stability.

\section{ACM Classification Keywords}

I.6.3 SIMULATION AND MODELING: Applications

\section{INTRODUCTION}

Complex, intelligent, adaptive and autonomous systems and their components are object of research in many domains. In the recent books on Modeling and Simulation (M\&S) support for system engineering processes [1, 2], possible application domains identified are:

- Military and defense applications;

- Transportation and traffic applications;

- Space applications, including space-based communications;

- Energy, in particular sustainable energy applications;

SpringSim MSCIAAS 2016, April 3-6, 2016, Pasadena, CA, USA Copyright 2016 Society for Modeling \& Simulation International (SCS)
- Urban planning and control applications;

- Cyber security applications;

- Etc.

The topic of M\&S support for autonomous systems has also been addressed in two workshops organized by the NATO M\&S Center of Excellence, both proceedings have been published and are publically available [3, 4]. In most of these application, the focus lies on the implementation of simulation solutions to provide decision support with the potential for automation in later phases. The use of agent based methods is most often recommended as the appropriate modeling paradigm for this kind of application. In every case described, the main motivation for using simulation was obtaining numerical insight into the dynamic behavior of the complex, intelligent, adaptive and autonomous systems and its components.

Naturally, the result is a large amount of data that needs to be analyzed, often using statistical methods. As featured in a recent article in the Nature magazine [5], blindly applying statistical cookbook solutions without understanding the application domain or the validity contexts of the methods can lead to insufficient or wrong interpretations. This is also true for the domain of complex, intelligent, adaptive and autonomous systems: if the analyst assumes the same behavior he is used to from traditional simulation system in which the simulated activities are not intelligent and adaptive, the results will be flawed.

The following sections of this paper will therefore address the questions each simulationists has to answer when using simulation to design, analyze, and control complex, intelligent, adaptive and autonomous systems and its components.

- How many solution clusters do I observe? Are there any bifurcations in my experiment? What does the solution space look like?

- How stable are my solutions? How are the individual results making up the cluster distributed? How big is the stochastic diversity of the solution?

- How sensitive are my solutions to slight variations in the initial conditions? What are the most important factors that have to be evaluated in more detail? 
The methods and heuristics described here are neither exclusive nor complete. However, they show that following statistical procedures blindly without applying domain knowledge of the supported domain as well as implementation logic of the supporting system can lead to misleading results and wrong interpretations.

\section{CHARACTERISTICS OF ENTITIES}

The simulated individual entities within complex, intelligent, adaptive and autonomous systems differ from components in centrally organized systems. It is worth to analyze the terms in regard to these characteristics:

- Complex: There is no agreed to definition of complexity, but there is some agreement that complexity describes a system comprising many various elements with many different forms of relations utilizing multiple interfaces supporting often non-linear interactions leading to holistic and emergent behaviors.

- Intelligent: In the contexts of artificial and computational systems, intelligence normally refers to the ability of sense-making and decision-making. Sense-making recognizes the need for actions based on the current perception, and decision-making selects the best action possible to pursue a goal-directed behavior.

- Adaptive: This is the ability to change to fit better for some purpose or situation, often also understood as the ability to learn. The entities must be able to dynamically change their rules to adapt their behavior to new constraints and situations.

- Autonomous: This is the ability of a system to act on its own goals, its perceptions, and its knowledge without any outside intervention. This does not exclude communication with other entities or humans, but the system acts on this information, not as a remote controlled entity.

The entities within our domain of interest are therefore best understood as agents that perceive their environment through a sensory system, make sense of the perception and make a decision what action to conduct, communicate with other entities in these processes, conduct the action and observe if the result was as expected, and adapt their behavior to new situations and constraints, to either pursue a goal-directed behavior or simply sustainment of the complex system.

The intelligent and adaptive characteristics ensure that the simulated entities observe the situated environment for possibilities to accomplish their goals. They take advantage of situations and exploiting weaknesses they perceive. They can follow overarching orders, but each individual entity still acts autonomously in following these directions.

These characteristics result in emergent effects for the system comprising of these entities that require an adequate use of statistics to gain the correct insights.

\section{NUMBER OF SOLUTIONS}

Jakob Bernoulli proved the law of large numbers in statistics, which states that, as the number of identically distributed, randomly generated variables increases, their sample mean approaches their theoretical mean. Furthermore, the central limit theorem (CLT) states that with the growing sample size the distribution becomes more like the normal distribution of the entire population. The power of the CLT is that it applies starting from any example of observations of instantiations of the experiments with essentially any distribution. It is therefore standard practice to assume that complex, intelligent, adaptive and autonomous systems will also follow these insights and can be evaluated using the CLT.

This, however, requires to make the assumption that the random experiments leading to the instantiations of the observed experiments are independent and leading to one distribution, which means one main course of action with an assumed mean value around which the observations are distributed within a certain variance.

This assumption is often not valid due to scenario constraints: Assume the simulation of traffic following the streets within a city. At every crossing, cars can decide which way they follow, resulting in a bifurcation in the model. As long as all forks are joining later, and all possible ways are similar, we can observe similar results meeting the assumptions. But what happens if you only have two alternative routes, and on one route, you have construction going on? If your measure of performance is the time needed to get from the starting point to the end point, you will have two mean values in this example: the first one for cars taking the unhindered route, the second one for the cars being slowed down by the construction. The bifurcation in the scenario leads to cluster building in the results. For each cluster, the CLT can be applied, but not to the overall result. In practice, rare but plausible results are often marked as outliers and are not taken into account for the evaluation process. Instead of adjusting our evaluation to our observations, we adjust our observations to justify the evaluation. This is bad practice, in particular in the context of this paper.

This structural effect is reinforced within complex, intelligent, adaptive and autonomous systems, as the smart entities take advantage of changes in the situation, creating additional bifurcation points resulting in structural changes resulting from random effects. If a random effect leads to an advantage for the simulated smart entities, they will perceive this new constraint and act accordingly. In the traffic example given above, the current speed possible for alternative routes may be derived by a random experiment: on average, this street allows you to drive $42 \mathrm{mph}$, but there is a variance of $5 \mathrm{mph}$. If a streets receives a high speed rate due to the random experiment, the smart entities will take advantage of this and shift the observed values accordingly. If the complex, intelligent, adaptive and autonomous system comprises of rivaling groups in which both groups are smart, 
the effect will reduce the overall variance as discussed in the next section.

As a general observation, bifurcation is not the exception but the rule in complex, intelligent, adaptive and autonomous systems. This has consequences for the design of experiments as well as for the choice of evaluation of results. We cannot assume that the resulting distribution is a standard distribution with one mean value and respective variance. Instead, there are likely several possible means with variances that are composed to the generated to solution space. As a rule, there is not a single solution, but a multitude of them. Alternative solutions are not outliers that can safely be ignored, but special cases that deserve special attention.

In order to identify these multiple solutions and separate the observations accordingly, multivariate statistics [6] can be applied, in particular cluster analysis. In general, cluster analysis identifies groups of objects that belong together. Applied in this context, it allows the separation of observations which belong to the various solutions distributed in the solution space due to bifurcation as described above. The following figure shows a solution space defined by two parameters that exposes three solutions.

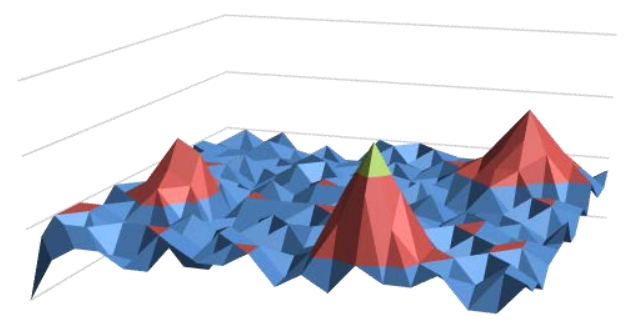

Figure 1. Solution Space with three Solutions.

In particular when interested in optimization, these characteristics become interesting as well, in particular when the possible solutions are too huge to allow for a complete computation of the solution space. In this case, a heuristic is needed to ensure that at least the interesting areas in the solution space are found.

The individual interesting solutions are usually connected with local extreme values. As the solution space, defined by the multitude of free parameters that usually are the input parameters for the system, is often huge, an efficient way to scan through possible solutions is needed. The domain of artificial intelligence developed several optimization heuristics [7] that can be applied in this context. Many of these methods have been recently rediscovered in the context of Deep Learning.

In order to scan through a wide solution space and touch as many areas as possible, genetic algorithms have been proven to be useful, as they allow for randomly chosen trials that have been shown to lead to better solutions in hyperparameter optimizations than grid-searches or manual searches [8].

It is good practice to use the mutation rate as a parameter to support wide range of changes in the beginning, to scan through as many areas as possible first, and then reduce the mutation rate to closer evaluate most promising areas. The result should be getting a better understanding on the topology of the solution space with a clear hint to where to look for local extreme values.

Once the area to look for local extreme values is known, single-path optimization algorithms - like tabu search or simulated annealing - can be applied [7]. They deliver good results when starting relatively close to the optimal search value, which has been accomplished by the application of genetic algorithms to scan the whole solution space first.

It should be pointed out that finding all local extreme values is not guaranteed, as these algorithms are heuristics. Nonetheless, there is a high likelihood that even with many degrees of free parameters many solutions can be found. These technologies have been successfully applied in many optimization studies. As a concluding recommendation of this section, cluster analysis, genetic algorithms, and simulated annealing should belong into every tool set used for evaluation of complex, intelligent, adaptive and autonomous systems.

A clear understanding of the topology of the solution space is pivotal for understanding the complex system under evaluation. But knowing the surface is not enough. For each identified solution, its statistical stability and sensitivity need to be evaluated as well. They will be discussed in the following sections.

\section{STABILITY OF SOLUTIONS}

Although deterministic complex, intelligent, adaptive and autonomous systems are possible, it is highly likely that uncertainty and vagueness in data and processes will be captured in stochastic approximations. We already introduced the notion of the result of a random experiment before, but it is worth to mention in the context of our discussion of the stability of solutions it is pivotal to understand that each observation is only one incarnation of many possible outcomes of the experiment. Even if all input parameters for the system that initialize constraints of the environment and behavior of the entities are fixed, the observed result will differ. After observing a sufficient number of repetitions, we can derive a distribution of these results, allowing us to predict future outcomes within these constraints. But the simulationist generally faces the question: How many repetitions are needed to be statistically significant? The answer is: it depends!

The complexity of the system drives the complexity of the results. In order to know that enough repetitions have been collected, the resulting distributions before and after an additional result was obtained should be identical. As long as 
the distributions before and after the new result was added differ, we still found significant new information. Only if new results no longer change the distribution, enough quasiempirical evidence has been collected to be statistically significant.

The two tests that traditionally are conducted to analyze the equivalency of two distributions are the Z-test and the Kolmogorov-Smirnov test. Recently, the Epps-Singleton test has been introduced to determine whether two samples have been drawn from the same population [9]. For the evaluation of complex, intelligent, adaptive and autonomous systems it is therefore good practice to conduct such test to determine if enough observations have been collected to make statistically significant statements on the distribution of results. This is only the case if adding new observations to the distribution doesn't change the characteristics of the distribution. Rules of thumb like "20 runs are sufficient for practical purposes" are neither justified nor helpful in complex systems. Furthermore, it is also possible that in some regions of the solution space only a few repetitions are needed while in other regions many replications are necessary to gain significant insights. Conducting the tests only for one region and then assume that the result is applicable for the whole solution space is scientifically naïve or even fraudulent. Vaux [10] shows the overall need to better understand the application of statistics to avoid "sloppy science."

It is also pivotal to visualize the results accordingly. It is often not deeper understood practice to compute the mean value and its variance without analyzing the underlying observations further. The problem that bifurcations can result in more than one cluster has already been addressed. There are many interesting visualization methods available, and at least the "boxes and whiskers" approach should be used. In this approach, the individual observations are aggregated into the mean value, the median value, the standard variation, and a box that captures the middle $50 \%$ of the observations as shown in the following figure.

The 30 observations resulting from 30 repetitions under identical initial conditions are enumerated on the right and plotted as circles as a reference. The box with mean, median, and standard derivation computed for this examples are shown on the left. Even without knowing anything about the individual observations it is clear that this is not a standard distribution, as the $50 \%$ box has the mean value as an upper limit, and mean and median are relative far apart. Looking at the distribution this seems to be the result of two cluster with center close to 11 and 35, which are likely the result of a bifurcating event. The aggregated display gives at least a first hint at the distribution of the observation and the overall stability of the solution.

These few examples demonstrate already that looking at the mean value is not sufficient, but it is good practice to use the statistical stability in regard to choosing good solutions. Solutions with very different distributions can easily look very similar when only the aggregates are evaluated. The solution space shown in Figure 1 only displays one aggregated values. What actually is needed is an extension of each value as of this surface to be extended using the insights shown for a single solution in Figure 2. Which visualization is the best to use is topic of ongoing research, such as described in [11].

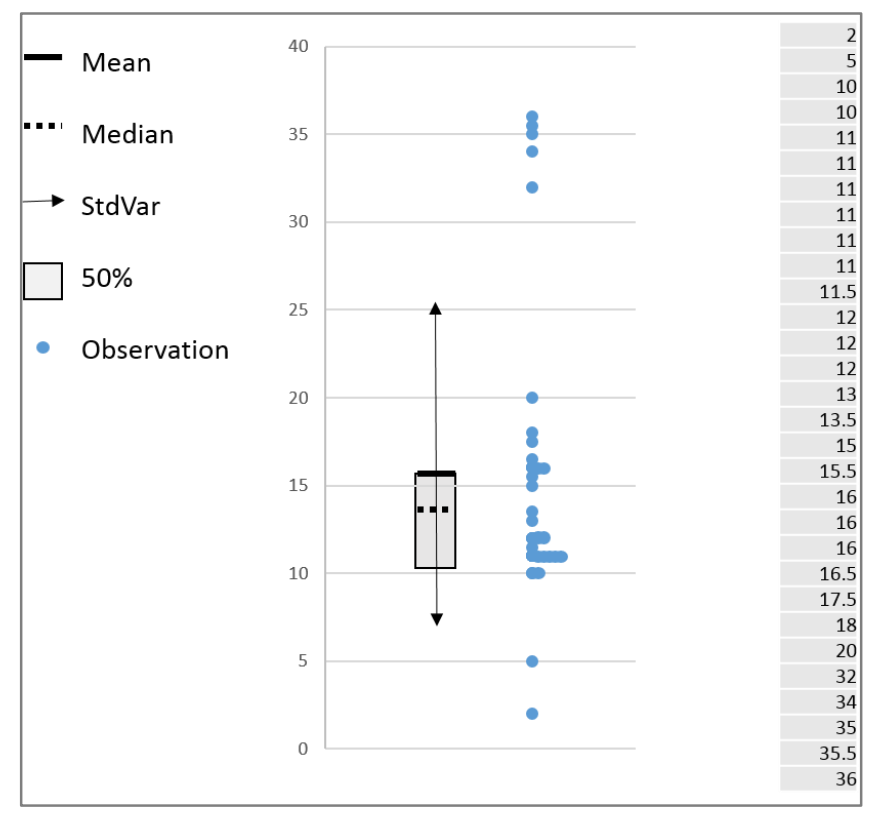

Figure 2. Box and Whiskers showing Stability of a Solution

Another aspect to be considered are the results presented in [12]: The following figure shows the results of applying heuristic optimization methods to 160 sensitive control variables (out of a total of 500) to improve the command and control for a combat scenario.

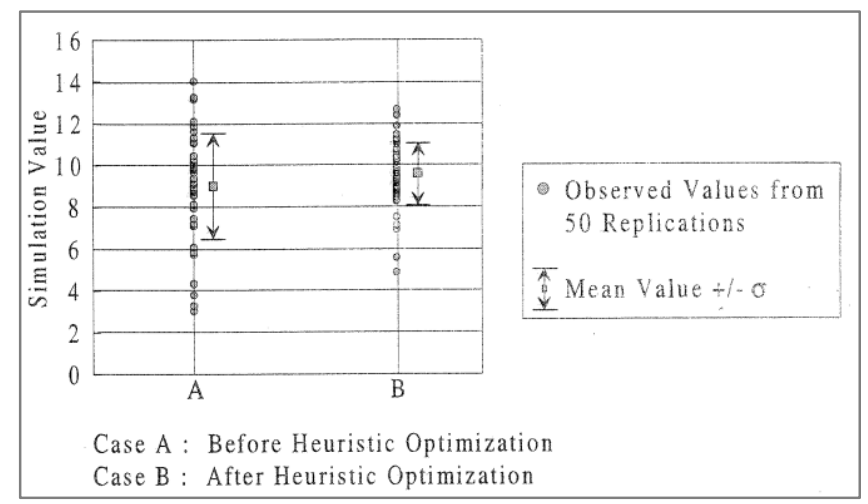

Figure 3. Simulation Results before and after heuristic optimization of rule sets for both antagonists [12, p. 17.9].

By improving the attacking as well as the defending side by making their command and control effective and robust, the stochastic dispersion was significantly reduced by eliminating inefficient edges of the decision tree. The effect 
could be observed in several thousand runs conducted in support of the NATO study.

When evaluating alternatives, they may expose valuable and interesting differences even if the mean value only changes slightly. In complex, intelligent, adaptive and autonomous systems it is therefore essential to understand these possibilities.

\section{SENSITIVITY OF SOLUTIONS}

If the solution space is completely understood and can be completely solved computationally, sensitivity analysis is already applied implicitly, as neighbored solutions are part of the complete picture. However, as the solution space in complex systems is usually too big to be computed completely - due to the combinatorically explosion of possible solutions due to the high number of parameters and their multiple nonlinear relations -, as a rule the simulationists will have to evaluate a set of solutions that often have been discovered by the application of heuristic methods. In other cases, subject matter experts may have come up with a best guess solution that needs to be evaluated. Also, a real world solution may be used to evaluate their applicability and sufficiency of provided capabilities for new constraints. In all these cases, the simulationists receives a set of solutions to be evaluated and often compared regarding their efficiency. Whenever this is the case, sensitivity analysis needs to be conducted in addition to the statistical stability analysis. Sensitivity evaluates the dependence of the solution from slight variations in the initial conditions.

In a deterministic setting, the same initial conditions always lead to the same results. It is therefore often assumed that similar initial conditions will also lead to similar results, but this assumption is often not correct. Non-linear function can lead to very different results for similar initial conditions, and it has been shown that non-linear functions behave chaotically when they are bounded and folded back into the defining interval, such as it is the case with the logistic function or the functions describing a double rod pendulum. Even if the initial conditions are arbitrarily close to each other, the results will be far apart after some iterations.

It is worth mentioning that when we are using computers to represent complex, intelligent, adaptive and autonomous systems, the predictability of our projections is significantly limited by these mathematical constraints: due to rounding errors inevitably connected with digital, discrete computing we can never make long term projections for systems comprising chaotic functions.

But even if no mathematical chaos is present in the system, nonlinearity can result in widely diverging results for similar initial conditions. As it is often likely that implementations vary slightly from the identified and recommended solution, sensitivity analysis is conducted to ensure that the result does not differ too much from the intended objectives. The recommended solution should result in similar performance for only slightly modified instantiations, and if this is not the case, the decision maker shall be made aware of this danger.
As the amount of free parameters is often too big to allow for a full combinatorial evaluation of neighbored initial solutions, the questions arises which parameters are the important ones and should be evaluated. This question is answered by factor analysis [6]. It is therefore good practice for the evaluation of solutions in complex systems to first apply factor analysis to identify the significant parameters and then conduct sensitivity analysis with such identified parameters for the metrics of interest.

Based on the observations in the earlier as well as in this section, the best solution for practical applications is not always the one with the highest expect mean value:

- If a solution is a peak in comparison with its neighbors, a slight variation in the solution can lead to a significant decrease in effectiveness. Even if the solution itself is superior, for practical reasons it may not be selected.

- If a solution has a high expected value, but the stochastic diversity is high/statistical stability is low, the likelihood to end up with a less capable solution due to stochastic effects is high. It may be better to choose a solution with more stability.

- The stochastic diversity/statistical stability of the neighbored solutions can be as important as the expected value. If the solution should be in a stable region with sufficient effectiveness, this may influence the choice of the solution to implement.

The following figure exemplifies these cases. Very often it is more important to reach a sufficient efficiency level for sure than to go for a risky optimal solution. In the left example, solution a7 may be preferable to a3, as a slight variation results in a worse outcome. In the middle example, solution b1 has a lower mean value, but the secure output is higher than b2, so it may be the preferred solution. In the right example, all mean values are identical, so that the variance will determine which solutions will be picked, depending if the decision maker is risk averse or not.

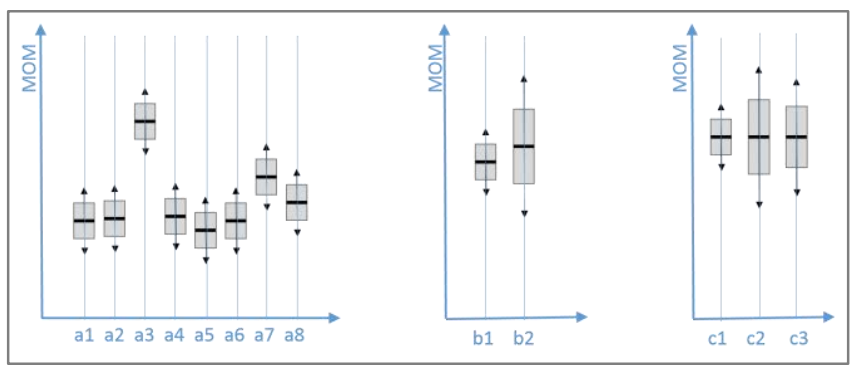

Figure 4. Examples for solutions.

It should be pointed out that the simulated intelligent components have to base their decisions on similar evaluations of their perceived situation. After they created a perception based on their sensor capabilities and made sense of their observation, the decision making processes have to evaluate their options and select their solution 


\section{CONSISTENCY OF INTERNAL AND EXTERNAL EVALUATION LOGIC}

The effect of structural variances is known for several decades and was first published in [13] and defined as follows: "Structural variance is the term applied to a discontinuity in results which were produced by smooth changes in an input parameter. It is caused by the structure of the model rather than by an error in the model, data, interactions represented or by any stochastic processes."

These discontinuities are always observed when the internal decision logic used by the smart components are unaligned with the evaluation logic used when computing the applied measure of merit (MOM). The resolution of the modeled entities that can be observed by the entities in their perception and that also are used by the evaluation processes to compute the metrics is the third important factor that needs to be aligned with the internal decision logic as well as with the external evaluation logic: whenever a level of detail in the system is used for a decision by the internal logic but is ignored in the evaluation of the results - or vice versa structural variances in form of discontinuities are likely to occur. The following figure shows the three components of the resulting harmonization and alignment principle.

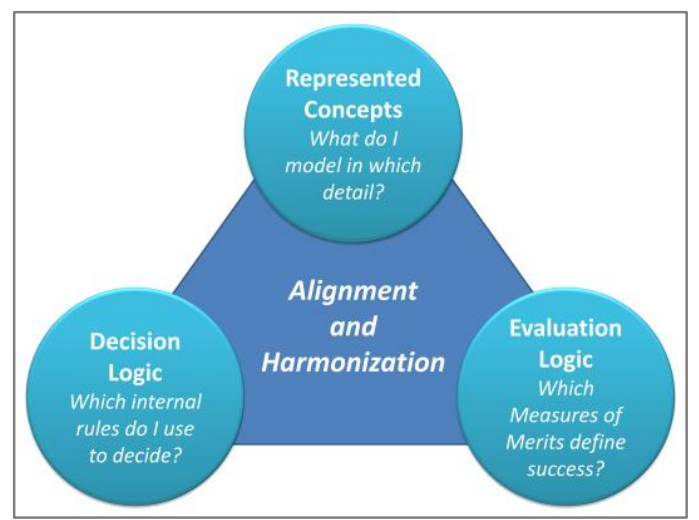

Figure 5. Harmonization and alignment principle [14, p. 81].

In order to follow the harmonization of processes and alignment of data principle, the rules and behavior of the smart components need to be transparent to a certain degree. The "correctness" of choices by the internal decision logic is determined by the external evaluation logic. In order to do so, the dimensional parameters used within the measures of merit to make the decisions and evaluate the results need to be aligned, otherwise discontinuities will be observed.

The following example from the defense domain shall explain this effect in more detail: the outcome of a battle is calculated by the reduction of the enemy forces. The simulated smart entities first defend in a forward position to give the main forces some time to prepare the main defense position before they fall back and become the reserve. While this behavior is very realistic, the applied MOM doesn't take all aspects not reflected in more attrition into account.
In complex, intelligent, adaptive and autonomous systems, these effects are likely to be observed. The simulationists must be aware of these relations to avoid misinterpretation of the results that may be based on insufficiently harmonized evaluation processes or misaligned data used as dimensional parameters.

\section{SUMMARY AND DISCUSSION}

The simulation support for the design and the evaluation of complex, intelligent, adaptive and autonomous systems has been identified as a candidate for a national research agenda [15]. It has many challenges that bring together the various fields of $M \& S$ research, reaching back some decades, but also some only addressed recently.

Of particular interest is the use of statistics in support of the evaluation. Many scientist use the means of statistics without full understanding of all assumptions and constraints, often leading to misperceptions and wrong interpretations. The simulationist dealing with these systems faces the same danger. The paper introduces at least some good practices for the evaluation of complex, intelligent, adaptive and autonomous systems:

- Do not assume a normal distribution! Conduct cluster analysis to identify if any bifurcation events did lead to a result in which several alternative courses of actions have to be evaluated.

- Do not trust point solutions! Evaluation the stochastic diversity of the solution. Do this for each cluster that contributes to the solution.

- Conduct a sensitivity analysis! Small changes in the initial conditions may result in significant changes in the result. This is true for the expected result as well as for the stochastic diversity.

Optimization of antagonist elements within the system may lead to a reduction of the stochastic diversity. However, it is of utter importance that the represented properties used as dimensional parameters in the metrics to make decisions in the smart entities are aligned with those used in the MOM to evaluate the overall solution for the system. Also, the internal decision processes and external evaluation processes must be aligned to avoid structural variances.

Another topic of interest in complex, intelligent, adaptive, and autonomous system is emergence. Although not addressed in detail, the heuristics and methods discussed in this paper are foundations to discover emergence. The "emergence complexity cone" introduced in [16] and shown in the following figure. For a detailed discussion and definition of the terms, the reader is referred to the original paper. Many principles addressed in [16] are also supported in this paper, as both are rooted in cybernetic principles and go back to control theoretic foundations.

However, even without an in depth discussion, the figure exemplifies the dimensions of emergency challenge that ultimately have to be addressed and that can structure a research agenda. The cone is linking emergent behavior 
taxonomy in increasing complexity on the y-axis and a possible categorization of stochastic and deterministic search-spaces on the $\mathrm{x}$-axis. The resulting cone volume depicts the variety, the perimeter as constraints, and the knowledge boundary as a cylinder that addresses the variety and constraints. The knowledge cylinder around simple and weak emergence in the deterministic domain signifies ample knowledge available to develop abstractions.

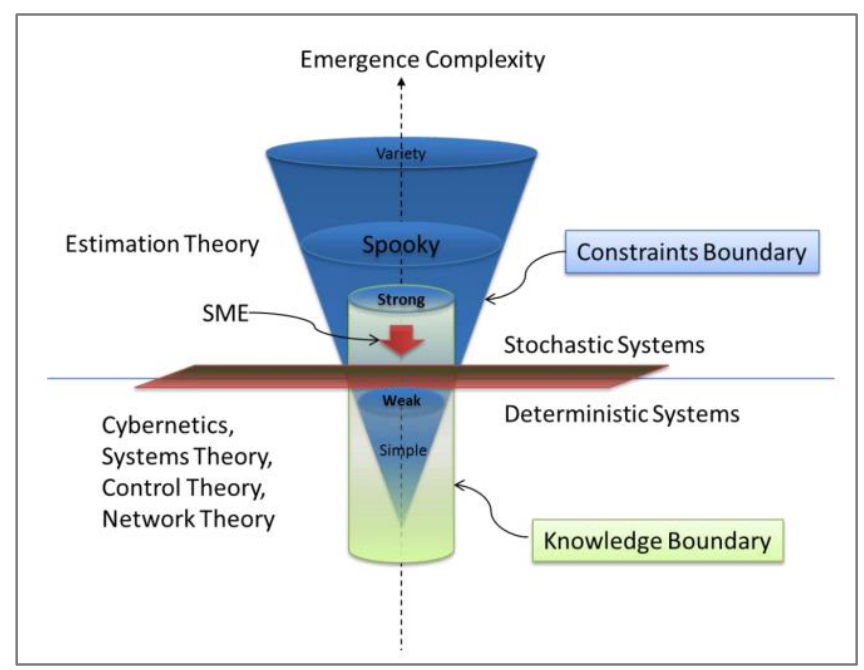

Figure 6. Emergence Complexity Cone [16].

The simulationists must be aware of these challenges as well as of possible solutions. Currently, the author is not aware of any analysis frameworks that support all these aspects sufficiently and hopes that this paper may spawn the development of such a framework that supports the experts as well as decision makers that are new to this domain.

\section{DISCLAIMER}

The author's affiliation with The MITRE Corporation is provided for identification purposes only, and is not intended to convey or imply MITRE's concurrence with, or support for, the positions, opinions or viewpoints expressed by the author.

\section{Approved for Public Release; Distribution Unlimited. Case Number 16-0437 \\ REFERENCES}

1. Gianni, D., D'Ambrogio, A., \& Tolk, A. (Eds.). Modeling and Simulation-Based Systems Engineering Handbook. CRC Press, Boca Raton, FL, USA, 2014.

2. Rainey, L. B., \& Tolk, A. (Eds.). Modeling and Simulation Support for System of Systems Engineering Applications. John Wiley \& Sons, Hoboken, NJ, USA, 2015.

3. Hodicky, J. (Ed.). Modelling and Simulation for Autonomous Systems: First International Workshop, MESAS 2014, Rome, Italy, May 5-6, 2014, Revised
Selected Papers (Vol. 8906). Springer, Heidelberg, Germany, 2014.

4. Hodicky, J. (Ed.). Modelling and Simulation for Autonomous Systems: Second International Workshop, MESAS 2015, Prague, Czech Republic, April 29-30, 2015, Revised Selected Papers (Vol. 9055). Heidelberg, Germany, 2015.

5. Nuzzo, R. Statistical errors. Nature 506, 7487 (2014), 150-152.

6. Afifi, A., May, S., \& Clark, V.A. Practical Multivariate Analysis, $5^{\text {th }}$ Ed. Chapman and Hall/CRC, Boca Raton, FL, USA, 2011.

7. Pham, D., \& Karaboga, D. Intelligent Optimization Techniques: Genetic Algorithms, Tabu Search, Simulated Annealing, and Neural Networks. Springer Science \& Business Media, New York, NY, USA, 2012.

8. Bergstra, J., \& Bengio, Y. Random search for hyperparameter optimization. The Journal of Machine Learning Research, 13, 1 (2012), 281-305.

9. Goerg, S. J., Kaiser, J., \& Bundesbank, D. Nonparametric testing of distributions - The EppsSingleton two-sample test using the empirical characteristic function. Stata Journal, 9, 3 (2009), 454.

10. Vaux, D. L. Research methods: Know when your numbers are significant. Nature 492, 7428 (2012), 180181.

11. Collins, A. J., Knowles Ball, D’A., Romberger, J. A Discussion on Simulations' Visualization Usage. Proceedings of the Winter Simulation Conference, Huntington Beach, VA, IEEE Press (2015), 2827-2835.

12. Hofmann, H., Schnurer, R., \& Tolk, A. On the Impact of Stochastic Modeling in a Rule Oriented Combat Simulation Model on Division/Corps Level. NATO Technical Proceedings AC/243(Panel 7)TP/9, Brussels, Belgium (1995), 17.1-17.12.

13. Hawkins, G. Structural variance and other related topics in the SHAPE Armour/Anti-Armour study. In Huber, R.K. (Ed.): Systems Analysis and Modeling in Defense Development, Trends, and Issues, Plenum Press, New York, NY, 1984.

14. Tolk, A. Engineering Principles for Combat Modeling and Distributed Simulation. John Wiley \& Sons, Hoboken, NJ, 2012.

15. Tolk, A., Balci, O., Combs, C.D., Fujimoto, R., Macal, C. M., Nelson, B. L., \& Zimmerman, P. Do we need a national research agenda for modeling \& simulation? Proceedings of the Winter Simulation Conference, Huntington Beach, CA, IEEE Press (2015), 2571-2585.

16. Mittal, S., Rainey, L. Harnessing Emergence: The design and control of emergent behavior in system of systems engineering. Proceedings of the Summer Simulation Multi-Conference, Chicago, IL, 2014. 